\title{
COUNTEREXAMPLES TO LOCAL EXISTENCE FOR QUASILINEAR WAVE EQUATIONS
}

\section{HANS LINDBLAD}

\section{Introduction and the main argument}

In this paper, we study quasilinear wave equations and ask how regular the initial data must be to ensure that a local solution exists. We present counterexamples to local existence for typical model equations. We also give the proof in the quasilinear case as well. The proof for the semilinear case was given in Lindblad $[7,9]$. The semilinear case counter examples are sharp, in the sense that for initial data with slightly more regularity, a local solution exists. This was shown recently in Klainerman-Machedon [3-5], Ponce-Sideris [11] and Lindblad-Sogge [10] using space time estimates and generalizations of these estimates known as Strichartz' estimates. For quasilinear equations, however, the optimal result is still unknown; there is a gap between the counterexamples we present here and a recent improvement on the local existence result by Tataru[15] and BahouriChemin [1]. Consider the Cauchy problem for a quasilinear wave equation:

$$
\begin{gathered}
\sum_{i, j=0}^{3} g^{i j}\left(u, u^{\prime}\right) \partial_{x^{i}} \partial_{x^{j}} u=F\left(u, u^{\prime}\right), \quad(t, x) \in S_{T}=[0, T) \times \mathbb{R}^{3}, \\
u(0, x)=f(x), \quad u_{t}(0, x)=g(x),
\end{gathered}
$$

where $\partial_{x^{0}}=\partial_{t}, g^{i j}$, and $F$ are smooth functions of $u$ and its first order derivatives. We also assume that $\left\{g^{i j}\right\}_{i, j=0}^{3}$ is a symmetric matrix that is close to the coefficient matrix of $\square=\partial_{t}^{2}-\sum_{i=1}^{3} \partial_{x^{i}}^{2}$;

$$
\sum_{j, k=0}^{3}\left|g^{j k}-m^{j k}\right| \leq 1 / 2, \quad \text { where }\left\{\begin{array}{l}
m^{00}=1, \quad m^{j j}=-1, j>0 \\
m^{j k}=0, \quad \text { if } j \neq k,
\end{array}\right.
$$

so that (1.1) is hyperbolic. Let $\dot{H}^{\gamma}$ denote the homogeneous Sobolev space with norm $\|f\|_{\dot{H}^{\gamma}}=\left\|\left|D_{x}\right|^{\gamma} f\right\|_{L^{2}}$ where $\left|D_{x}\right|=\sqrt{-\Delta_{x}}$ and set

$$
\|\left. u(t, \cdot)\right|_{\gamma} ^{2}=\int\left(\left.\left.|| D_{x}\right|^{\gamma-1} u_{t}(t, x)\right|^{2}+\left.\left.|| D_{x}\right|^{\gamma} u(t, x)\right|^{2}\right) d x
$$

Received August 31, 1998.

The author was supported in part by the National Science Foundation. 
Since the norms (1.3) are more or less the only norms that are preserved for the linear wave equation $\square u=0$, it is natural to look for existence in the spaces associated with these norms. We want to find the smallest possible $\gamma$ such that

$$
\begin{aligned}
& (f, g) \in \dot{H}^{\gamma}\left(\mathbb{R}^{3}\right) \times \dot{H}^{\gamma-1}\left(\mathbb{R}^{3}\right), \\
& \operatorname{supp} f \cup \operatorname{supp} g \subset\{x ;|x| \leq R\},
\end{aligned}
$$

implies that we have a local distributional solution of (1.1) for some $T>0$, satisfying

$$
\left(u, \partial_{t} u\right) \in C\left([0, T] ; \dot{H}^{\gamma}\left(\mathbb{R}^{3}\right) \times \dot{H}^{\gamma-1}\left(\mathbb{R}^{3}\right)\right) .
$$

We say that $u$ is in $\mathbf{H}^{\gamma}[0, T]$ if $\left(u, \partial_{t} u\right) \in L^{\infty}\left([0, T] ; \dot{H}^{\gamma}\left(\mathbb{R}^{3}\right) \times \dot{H}^{\gamma-1}\left(\mathbb{R}^{3}\right)\right.$, (i.e., if the norm (1.3) is uniformly bounded on $[0, T])$. Similarly we say that data $(f, g)$ is in $\mathbf{H}^{\gamma}[0]$ if $(f, g) \in \dot{H}^{\gamma}\left(\mathbb{R}^{3}\right) \times \dot{H}^{\gamma-1}\left(\mathbb{R}^{3}\right)$, (i.e., if the norm (1.3) is bounded initially when $t=0$ ).

To avoid certain peculiarities concerning non-uniqueness we also require that $u$ is a proper solution:

Definition 1.1. We say that $u$ is a proper solution of (1.1) if it is a distributional solution, and if $u$ is the weak limit of a sequence of smooth solutions $u_{\varepsilon}$ to (1.1) with data $\left(\phi_{\varepsilon} * f, \phi_{\varepsilon} * g\right)$, where $\phi_{\varepsilon}(x)=\phi(x / \varepsilon) \varepsilon^{-n}$ for some function $\phi$ satisfying $\phi \in C_{0}^{\infty}, \int \phi d x=1$.

Even if one has smooth data and hence a smooth solution there might still be another distributional solution which satisfies initial data in the space given by the norm (1.3). In fact, $u(t, x)=\sqrt{2} H(t-|x|) / t$ satisfies $\square u=u^{3}$ in the sense of distribution theory. If $\gamma<1 / 2$ then $\|u(t, \cdot)\|_{\gamma} \rightarrow 0$ when $t \rightarrow 0$ by homogeneity. Since $u(t, x)=0$ is another solution with the same data it follows that we have non-uniqueness in the class (1.6) if $\gamma<1 / 2$. Definition 1.1 picks out the smooth solution if there is one.

Our main theorem is the following:

Theorem 1.2. Consider the Cauchy problem in $\mathbb{R}^{1+3}$;

$$
\begin{array}{r}
\square u=\left(D^{\ell} u\right) D^{k-\ell} u, \quad D=\left(\partial_{x^{1}}-\partial_{t}\right), \\
u(0, x)=f(x), \quad u_{t}(0, x)=g(x),
\end{array}
$$

where $0 \leq \ell \leq k-\ell \leq 2$, $\ell=0,1$. Let $\gamma=k$. Then there exists data $(f, g)$ satisfying (1.4)-(1.5), with $\|f\|_{\dot{H}^{\gamma}}+\|g\|_{\dot{H}^{\gamma-1}}$ arbitrarily small, such that (1.7) does not have any proper solution satisfying (1.6) in $S_{T}=[0, T) \times \mathbb{R}^{3}$ for any $T>0$.

Remark 1.3. It also follows from the proof of the theorem that either we have non-existence of any distributional solution satisfying (1.6) or else we have nonuniqueness in a domain of dependence in this class (see Definition 1.6 and Theorem 1.8). Also, stronger statements hold in the different cases (see Lindblad[79].) I particular if $k-\ell=\ell=0$ then there is no distributional solution in $L^{2}\left(S_{T}\right)$. 
Remark 1.4. By a simple scaling argument one gets a counterexample to wellposedness, but it has lower regularity than our counterexamples: $\gamma<k-1 / 2$. Indeed, if $u$ is a solution of (1.7) which blows up when $t=T$ then $u_{\varepsilon}(t, x)=$ $\varepsilon^{k-2} u(t / \varepsilon, x / \varepsilon)$ is a solution of the same equation with lifespan $T_{\varepsilon}=\varepsilon T$ and $\left\|u_{\varepsilon}(0, \cdot)\right\|_{\gamma}=\varepsilon^{k-2+3 / 2-\gamma}\|u(0, \cdot)\|_{\gamma} \rightarrow 0$ if $\gamma<k-1 / 2$. By contrast, our counterexamples are designed to concentrate in one direction, close to a characteristic.

Remark 1.5. In Klainerman-Machedon[3-5] it was proved that for semilinear wave equations satisfying the "null condition" one can in fact get local existence for data having the regularity predicted by the scaling argument. Although the nonlinearities in our theorem have a special form the same result should hold for any quadratic form in the derivatives of $u$ that does not satisfy the "null condition".

Now, there is a unique way to write (1.7) in the form (1.1). In the semilinear case, $k-\ell \leq 1$, the equation is already in this form with $g^{j k}=m^{j k}$, where $m^{j k}$ is given by (1.2). In the quasilinear case, $k-\ell=2$,

$$
\sum_{i, j=0}^{3} g^{i j}(t, x) \partial_{x^{i}} \partial_{x^{j}}=\partial_{x^{0}}^{2}-\sum_{i=0}^{3} \partial_{x^{i}}^{2}-v(t, x)\left(\partial_{x^{0}}-\partial_{x^{1}}\right)^{2}, \quad \text { where } \quad v=D^{\ell} u
$$

We now define the notion of a domain of dependence.

Definition 1.6. Assume that $\Omega \subset \mathbb{R}_{+} \times \mathbb{R}^{3}$ is an open set equipped with a Lorentzian metric $g_{j k} \in C(\Omega)$ such that inverse $g^{j k}$ satisfies (1.2). Then $\Omega$ is said to be a domain of dependence for the metric $g_{i j}$ if the closure of the causal past $\Lambda_{t^{\prime}, x^{\prime}}$ of each point $\left(t^{\prime}, x^{\prime}\right) \in \Omega$ is contained in $\Omega$. Here $\Lambda_{t^{\prime}, x^{\prime}}$ is defined to be all points in $\Omega$ that can be joined to $\left(t^{\prime}, x^{\prime}\right)$ by a Lipschitz continuous curve $(t, x(t)) \in \Omega, t \leq t^{\prime}$, satisfying

$$
\sum_{i, j=0}^{3} g_{i j}(x) \frac{d x^{i}}{d t} \frac{d x^{j}}{d t} \geq 0, \quad x^{0}(t)=t
$$

almost everywhere. Since a solution $u$ to (1.1) gives rise to a unique metric $g_{j k}$ we say that $\Omega$ is a domain of dependence for the solution $u$ if it is a domain of dependence for $g_{j k}$.

The importance of the concept of domain of dependence is Huygens principle which says that changing the initial data outside the intersection of the domain of dependence with the inital plane $t=0$ should not change the solution in the domain of dependence, or in other words; information should not travel faster than the speed of light. This is true for smooth solutions. We also have uniqueness and continuous dependence of data for smooth solutions in a domain of dependence. Hence if there is a smooth solution in a domain of dependence it is unique within the class of proper solutions. 
Lemma 1.7. Suppose $u \in C^{\infty}(\Omega)$ is a solution to (1.7) in a domain of dependence $\Omega$ with initial data $(f, g)$ on $\Omega_{0}=\{x ;(0, x) \in \Omega\}$. In the quasilinear case, $k-\ell=2$, assume also that $\left\|D^{\ell} u\right\|_{L^{\infty}(\Omega)} \leq \delta$. Suppose that $u_{\varepsilon} \in C^{\infty}\left(S_{T}\right)$, where $S_{T}=[0, T) \times \mathbb{R}^{3}$, are solutions of $(1.7)$ with data $\left(f_{\varepsilon}, g_{\varepsilon}\right)$ where $\left(f_{\varepsilon}, g_{\varepsilon}\right) \rightarrow(f, g)$ in $C^{\infty}\left(\Omega_{0}\right)$. Then $u_{\varepsilon} \rightarrow u$ in $C^{\infty}\left(\Omega \cap S_{T}\right)$. Here $C^{\infty}(\mathcal{O})$ denotes the set of smooth function on $\mathcal{O}$ with the usual topology defined by the semi-norms $\rho_{\alpha, K}(f)=\sup _{x \in K}\left|\partial^{\alpha} f(x)\right|$, for $K$ a compact subset of $\mathcal{O}$.

The proof of Lemma 1.7 is standard so we postpone it to an appendix. It is essential that $\Omega$ is a domain of dependence for Lemma 1.7 to be true; one needs exactly this in order to be able to use the energy method to get estimates.

Theorem 1.8. There is an open set $\Omega \subset \mathbb{R}_{+} \times \mathbb{R}^{3}$ and a solution $u \in C^{\infty}(\Omega)$ of

$$
\square u=\left(D^{\ell} u\right) D^{k-\ell} u, \quad D=\left(\partial_{x^{1}}-\partial_{t}\right), \quad 0 \leq \ell \leq k-\ell \leq 2, \quad \ell=0,1,
$$

such that $\Omega$ is a domain of dependence for $u$ and writing

$$
\Omega_{t}=\{x ;(t, x) \in \Omega\},
$$

we have that $\partial \Omega_{0}$ is smooth,

$$
\sum_{|\beta| \leq k,\left|\beta_{0}\right| \leq 1} \int_{\Omega_{t}}\left(\partial^{\beta} u(t, x)\right)^{2} d x \quad \begin{cases}<\infty, & \text { when } t=0 \\ \infty, & \text { when } t>0\end{cases}
$$

where $\beta=\left(\beta_{0}, \ldots, \beta_{3}\right)$ and $\partial^{\beta}=\partial_{t}^{\beta_{0}} \cdots \partial_{x^{3}}^{\beta_{3}}$. In fact;

$$
\int_{\Omega_{t}}\left(D^{k-\ell} u(t, x)\right)^{2} d x=\infty, \quad t>0
$$

Furthermore, in the quasilinear case, $k-\ell=2$, we can pick $u$ with $\left\|D^{\ell} u\right\|_{L^{\infty}(\Omega)}$ arbitrarily small.

Proof of Theorem 1.2. By Theorem 1.8 we get a solution $\bar{u}$ in a domain of dependence $\Omega$ with initial data $\bar{u}(0, x) \in H^{k}\left(\Omega_{0}\right)$ and $\bar{u}_{t}(0, x) \in H^{k-1}\left(\Omega_{0}\right)$. We can extend these to $f \in H^{k}\left(\mathbb{R}^{3}\right)$ and $g \in H^{k-1}\left(\mathbb{R}^{3}\right)$, see Stein [14]. If there exist a proper solution $u$ of $(1.7)$ in $S_{T}=[0, T] \times \mathbb{R}^{3}$ with these data, it follows from Definition 1.1 and Lemma 1.7 that $u$ is equal to $\bar{u}$ in $S_{T} \cap \Omega$, contradicting (1.6).

Let us now briefly describe how to construct the solution $u$ and the domain of dependence $\Omega$ in Theorem 1.8. First we find a solution $u(t, x)=u_{1}\left(t, x^{1}\right)$, depending only on one space variable $x^{1} \in \mathbb{R}$, that develops a certain singularity for $t>0$ along a non timelike curve $x^{1}=\mu(t)$, (i.e., $\Omega^{1}=\left\{\left(t, x^{1}\right) ; x^{1}>\mu(t)\right\}$ is a domain of dependence). Then we will find a domain of dependence $\Omega \subset$ $\Omega^{1} \times \mathbb{R}^{2}$ for $u_{1}\left(t, x^{1}\right)$ such that the solution is in $H^{k}\left(\Omega_{0}\right)$ and such that the curve 
$x^{1}=\mu(t), x^{2}=x^{3}=0$, lies on $\partial \Omega$, which ensures that the solution is not in $H^{k}\left(\Omega_{t}\right)$ for $t>0$. This means that $\Omega$ contains the causal past, for the metric given by $u_{1}$, of the above curve for small $t$.

The equation $\square u=D^{\ell} u D^{k-\ell} u$, becomes

$$
D_{+} D_{-} u_{1}+D_{-}^{\ell} u_{1} D_{-}^{k-\ell} u_{1}=0, \quad \text { where } \quad D_{ \pm}=\partial_{x^{1}} \pm \partial_{t} .
$$

This equation can be solved by integrating along characteristics. By choosing particular initial data

$$
D_{-} u_{1}\left(0, x^{1}\right)=\chi^{(3-k)}\left(x^{1}\right), \quad D_{+} u_{1}\left(0, x^{1}\right)=0,
$$

$$
\text { where } \chi\left(x^{1}\right)=\int_{0}^{x^{1}}-\varepsilon|\log | s /\left.4\right|^{\alpha} d s, \quad 0<\alpha<\frac{1}{2}, \varepsilon>0,
$$

we get a solution

$$
u_{1} \in C^{\infty}\left(\Omega^{1}\right), \quad \text { where } \quad \Omega^{1}=\left\{\left(t, x^{1}\right) ; \mu(t)<x^{1}<2-t\right\} \subset \mathbb{R}_{+} \times \mathbb{R}^{1},
$$

for some function $\mu(t)$ with $\mu(0)=0$, such that $\Omega^{1}$ is a domain of dependence and such that $u_{1}\left(t, x^{1}\right)$ has a singularity along $x^{1}=\mu(t)$. One sees this from the solution formulas obtained from integrating along characteristics (see section 2). Essentially what is happening is that the initial data (1.13)-(1.14) has a singularity when $x^{1}=0$. For the linear equation, $u_{t t}-u_{x^{1} x^{1}}=0$, the singularity would just have propagated along a characteristic, however the nonlinearity causes the solution to increase and this strengthens the singularity for $t>0$. This is the same phenomena that causes blow-up for smooth initial data. The blow-up along characteristics occures at a time $\sim-1 / \chi^{\prime}$. Because $\chi^{\prime}(0+)=-\infty$, the blow-up happens directly close to $x^{1}=0$.

Define $\Omega \subset \mathbb{R}_{+} \times \mathbb{R}^{3}$ to be the largest domain of dependence for the metric obtained from the solution $u(t, x)=u_{1}\left(t, x^{1}\right)$ such that

$$
\Omega \subset \Omega^{1} \times \mathbb{R}^{2}, \quad \Omega_{0}=\{x ;(0, x) \in \Omega\}=B_{0}=\{x ;|x-(1,0,0)|<1\} .
$$

(It follows from Definition 1.6 that the union and intersection of a finite number of domains of dependence is a domain of dependence so indeed a maximal domain exists.) The initial data (1.13)-(1.14) was chosen so that (1.10) just is finite if $t=0$, an easy calculation shows that this is equivalent to $\int y \chi^{\prime \prime}(y)^{2} d y<\infty$.

Let $\Omega_{t}$ be as in (1.9) and

$$
S_{t}\left(x^{1}\right)=\left\{\left(x^{2}, x^{3}\right) \in \mathbb{R}^{2} ;\left(x^{1}, x^{2}, x^{3}\right) \in \Omega_{t}\right\}, \quad a_{t}\left(x^{1}\right)=\int_{S_{t}\left(x^{1}\right)} d x^{2} d x^{3} .
$$

With this notation the integral in (1.11) becomes, if we integrate out the $x^{2}$ and $x^{3}$ variables

$$
\int_{\mu(t)}^{2-t} a_{t}\left(x^{1}\right)\left(D_{-}^{k-\ell} u_{1}\left(t, x^{1}\right)\right)^{2} d x^{1}
$$


The proof that this integral is infinite consists of estimating the two factors in the integrand from below, close to $x^{1}=\mu(t)$.

In the semilinear case the metric $g^{j k}$ is just $m^{j k}$ so $\Omega^{1}$ is a domain of dependence if and only if $\mu^{\prime}(t) \geq 1$ and it follows that $\Omega=\Omega^{1} \times \mathbb{R}^{2} \cap \Lambda$, where $\Lambda=$ $\{(t, x) ;|x-(1,0,0)|+t<1\}$. Hence for $x^{1}>\mu(t) ; S_{t}\left(x^{1}\right)=\left\{\left(x^{2}, x^{3}\right) ;\left(x^{1}-1\right)^{2}+\right.$ $\left.x^{2}{ }^{2}+x^{3}{ }^{2}<(1-t)^{2}\right\}$ so then $a_{t}\left(x^{1}\right)=\pi\left(2-t-x^{1}\right)\left(x^{1}-t\right)$. Also, the specific solution formulas are relatively simple. In particular if $k-\ell=l=1$, then it is easy to verify that

$$
D_{-} u_{1}\left(t, x^{1}\right)=\frac{\chi^{\prime}\left(x^{1}-t\right)}{1+t \chi^{\prime}\left(x^{1}-t\right)}
$$

satisfies (1.12)-(1.13) when $1+t \chi^{\prime}\left(x^{1}-t\right)>0$. Since $\chi^{\prime}(0+)=-\infty$ and $\chi^{\prime \prime}>0$ it follows that there is a function $\mu(t)$, with $\mu^{\prime}(t)>1$ and $\mu(0)=0$, such that $1+t \chi^{\prime}\left(x^{1}-t\right)=0$, when $x^{1}=\mu(t)$. Hence $1+t \chi^{\prime}\left(x^{1}-t\right) \leq C(t)\left(x^{1}-\mu(t)\right)$ so

$$
\int_{\mu(t)}^{\frac{1}{2}} a_{t}\left(x^{1}\right)\left(D_{-} u_{1}\left(t, x^{1}\right)\right)^{2} d x^{1} \geq \int_{\mu(t)}^{\frac{1}{2}} \frac{\left(x^{1}-t\right) d x^{1}}{C(t)^{2}\left(x^{1}-\mu(t)\right)^{2}}=\infty .
$$

We refer the reader to Lindblad [9] for the complete proof in the semilinear case.

In this paper we will concentrate on the quasilinear case. Here, the solution formulas are less explicit and given in terms of the characteristics for the equation (1.12). In the quasilinear case, estimating $a_{t}\left(x^{1}\right)$ from below requires control of the geometry of the causal past for the metric whos inverse is given by

$$
g^{i j}\left(t, x^{1}\right) \partial_{x^{i}} \partial_{x^{j}}=\partial_{t}^{2}-\sum_{i=1}^{3} \partial_{x^{i}}^{2}-v\left(t, x^{1}\right)\left(\partial_{x^{1}}-\partial_{t}\right)^{2}, \text { where } v=\left(\partial_{x^{1}}-\partial_{t}\right)^{\ell} u_{1} \text {. }
$$

\section{The quasilinear case}

The quasilinear case of the equation in one space dimension (1.12) can be factored

$$
\begin{aligned}
\left(\left(\partial_{t}+\partial_{x^{1}}\right)+v\left(\partial_{x^{1}}-\partial_{t}\right)\right)\left(\partial_{x^{1}}-\partial_{t}\right) u & =0, \quad \text { where } \quad v=\left(\partial_{x^{1}}-\partial_{t}\right)^{\ell} u \\
u\left(0, x^{1}\right)=0, \quad \partial_{t} u\left(0, x^{1}\right) & =-\chi^{(1-\ell)}\left(x^{1}\right)
\end{aligned}
$$

Here $\ell=0,1$. This equation can be solved by integrating along the characteristics. (2.1) is equivalent to

$$
\left(\partial_{t}+\frac{1+v}{1-v} \partial_{x^{1}}\right)\left(\partial_{x^{1}}-\partial_{t}\right) u=0
$$


The characteristics are given by $x^{1}+t=$ constant respectively $x^{1}=\phi(t, y)$ where

$$
\frac{d}{d t} \phi(t, y)=\frac{1+v(t, \phi(t, y))}{1-v(t, \phi(t, y))}, \quad \phi(0, y)=y
$$

(2.3) says that $\left(\partial_{x^{1}}-\partial_{t}\right) u$ is constant along the characteristics (2.4) so

$$
\begin{aligned}
\left(\left(\partial_{x^{1}}-\partial_{t}\right) v\right)(t, \phi(t, y)) & =\chi^{\prime}(y), \quad v(0, y)=0, & & \text { if } \quad \ell=0 \\
v(t, \phi(t, y)) & =\chi(y), & & \text { if } \quad \ell=1 .
\end{aligned}
$$

Let $\mathbb{R}_{+}=(0, \infty), \overline{\mathbb{R}_{+}}=[0, \infty)$ and let $C^{\ell} \cap \overline{C^{k}}(\Omega)=C^{\ell}(\Omega) \cap \overline{C^{k}}(\Omega)$, where $\overline{C^{k}}(\Omega)=C^{k}(\bar{\Omega})$. We have

Proposition 2.1. Let $0<\varepsilon<1$ and suppose that $\chi \in C^{\infty} \cap \overline{C^{0}}\left(\mathbb{R}_{+}\right)$and

$$
-\varepsilon<\chi<0, \quad \chi^{\prime}<0, \quad \chi^{\prime \prime}>0, \quad \chi(0)=0, \quad \chi^{\prime}(0+)=-\infty, \quad \chi^{\prime}(\infty)=0 .
$$

Then there are functions $\phi \in C^{\infty} \cap \bar{C}\left(\overline{\mathbb{R}_{+}} \times \mathbb{R}_{+}\right) \cap C^{1}\left(\overline{\mathbb{R}_{+}} \times \overline{\mathbb{R}_{+}} \backslash\{(0,0)\}\right)$, $\nu \in C^{\infty} \cap \bar{C}\left(\mathbb{R}_{+}\right)$and $v \in C^{\infty} \cap \bar{C}\left(\Omega^{1}\right)$, where

$$
\Omega^{1}=\left\{\left(t, x^{1}\right) ; x^{1}>\mu(t)=\phi(t, \nu(t)), t \geq 0\right\},
$$

such that (2.4)-(2.5) hold when $y>\nu(t), t \geq 0$, and

$$
\begin{gathered}
\nu(0)=0, \quad \nu^{\prime}(t) \geq 0, \\
\phi_{y}(t, y)>0, \quad y>\nu(t), \quad \phi_{y}(t, \nu(t))=0, \\
\left|v\left(t, x^{1}\right)\right|<\varepsilon, \quad\left(\partial_{x^{1}}-\partial_{t}\right) v\left(t, x^{1}\right)<0,
\end{gathered}
$$

when $t>0$ and $\left(t, x^{1}\right) \in \Omega^{1}$.

If $\ell=0$ then $\nu(t)=0$ and

$$
0<\phi_{y}(t, y)<e^{2 t \chi^{\prime}(y)} /(1-\varepsilon), \text { when } y>0 .
$$

If $\ell=1$, then $\nu^{\prime}(t)>0$ when $t>0$, and there is a function $M \in C\left(\mathbb{R}_{+}\right)$such that

$$
0<\phi_{y}(t, y)<M(t)(y-\nu(t)), \quad \text { when } \quad y>\nu(t), \quad t>0 \text {. }
$$

Remark. Note that $\Omega^{1}$ is a domain of dependence since $\nu^{\prime}(t) \geq 0$. Furthermore $\phi(t, \nu(t))=0$ so the function $v\left(t, x^{1}\right)$ defined by solving $x^{1}=\phi(t, y)$ for $y$ as a function of $\left(t, x^{1}\right)$ in (2.5) has a singularity along the curve $x^{1}=\mu(t)=\phi(t, \nu(t))$, which lies in $\partial \Omega^{1}$. 
The calculations simplify if we introduce new variables

$$
\begin{aligned}
& s=x^{1}+t, \quad q=x^{1}-t, \\
& U(s, q)=u((s-q) / 2,(s+q) / 2) .
\end{aligned}
$$

Then (2.1) becomes,

$$
\begin{aligned}
\left(\partial_{s}+V(s, q) \partial_{q}\right) \partial_{q} U(s, q)=0, & V(s, q)=\partial_{q}^{\ell} U(s, q) 2^{\ell} \\
U(y, y) & =0, \quad U_{q}(y, y)=\chi^{(1-\ell)}(y) 2^{-\ell}
\end{aligned}
$$

The characteristics for (2.12) are given by $s=$ constant respectively $q=h(s, y)$ where

$$
\frac{d}{d s} h(s, y)=V(s, h(s, y)), \quad h(y, y)=y
$$

By $(2.12), d U_{q}(s, h(s, y)) / d s=0$ so

$$
\begin{aligned}
V_{q}(s, h(s, y))= & \chi^{\prime}(y), \quad V(y, y)=0, \\
V(s, h(s, y))=\chi(y), & \text { if } \quad \ell=0,
\end{aligned}
$$

Proof of Proposition 2.1. We will first prove Proposition 2.1 in the case $l=0$. Differentiating (2.14) with respect to $y$ gives

$$
\frac{d}{d s} h_{y}(s, y)=V_{q}(s, h(s, y)) h_{y}(s, y), \quad h_{s}(y, y)+h_{y}(y, y)=1
$$

Since $h_{s}(y, y)=0$ by (2.14) and (2.13) it follows that

$$
\frac{d}{d s} h_{y}(s, y)=\chi^{\prime}(y) h_{y}(s, y), \quad h_{y}(y, y)=1
$$

which has the solution

$$
h_{y}(s, y)=e^{(s-y) \chi^{\prime}(y)}
$$

Since $h_{y}(s, y)>0$, when $y>0$, we can solve the equation $q=h(s, y)$ for $y$ as a function of $(s, q)$ so $(2.15)$ defines a function $U(s, q)=V(s, q)$, in

$$
\tilde{\Omega}_{1}=\{(s, q) ; q>h(s, 0)\},
$$

which satisfies (2.12)-(2.13). Furthermore by (2.15)

$$
V(s, h(s, y))=-\int_{y}^{s} V_{q}(s, h(s, z)) h_{y}(s, z) d z \leq-\int_{y}^{s} \chi^{\prime}(z) d z,
$$


since $\chi^{\prime}(z)<0$ so

$$
0 \leq V(s, h(s, y)) \leq \chi(y)-\chi(s)<\varepsilon<1, \quad \text { and } \quad V_{q}(s, h(s, y))<0 .
$$

We now want to change back to the original variables $\left(t, x^{1}\right)$. The characteristics $q=h(s, y)$ can be expressed as $x^{1}=\phi(t, y)$, if we introduce $t$ as a new parameter: $s=s(t, y)$ and set $\phi(t, y)=h(s, y)+t$. In fact, $t=(s-q) / 2=(s-h(s, y)) / 2$ so $d t / d s=(1-V(s, h(s, y)) / 2>0$. Since $h(\phi+t, y)=\phi-t$ we have

$$
\phi_{y}(t, y)=\frac{h_{y}(s, y)}{1-V(s, h(s, y))}>0, \quad \text { when } y>0,
$$

so, $u_{1}\left(t, x^{1}\right)=V\left(t+x^{1}, x^{1}-t\right)$ is a solution to $(2.1)-(2.2)$ in the set

$$
\Omega^{1}=\left\{\left(t, x^{1}\right) ; x^{1}>\mu(t)=\phi(t, 0)\right\} .
$$

Furthermore,

$$
s-y-2 t=h(s, y)-y=\int_{y}^{s} h_{s}(z, y) d z=\int_{y}^{s} V(z, h(s, y)) d z \geq 0,
$$

which in view of (2.18) and (2.17) proves (2.9).

Let us now deal with the case $\ell=1$, which in fact is much simpler. By (2.4) and $(2.5)$

so

$$
\frac{d}{d t} \phi(t, y)=\frac{1+\chi(y)}{1-\chi(y)}, \quad \phi(0, y)=y
$$

and

$$
\phi(t, y)=y+\frac{1+\chi(y)}{1-\chi(y)} t
$$

$$
\phi_{y}(t, y)=1+\frac{\chi^{\prime}(y)}{(1-\chi(y))^{2}} t .
$$

Since $\chi^{\prime}(0+)=-\infty$ and $\chi^{\prime}(\infty)=0$ it follows that there is a function $\nu(t)>0$, when $t>0$, such that

$$
\phi_{y}(t, \nu(t))=0, \quad \text { when } \quad t>0 .
$$

and $\nu(0+)=0$. We have

so

$$
\phi_{y y}(t, y)=\frac{(1-\chi(y)) \chi^{\prime \prime}(y)+2 \chi^{\prime}(y)^{2}}{(1-\chi(y))^{3}} t>0
$$

$$
0<\phi_{y}(t, y)<(y-\nu(t)) \sup _{z>\nu(t)} \phi_{y y}(t, z), \quad y \geq \nu(t)
$$

which proves (2.10). Furthermore

$$
0=\frac{d}{d t} \phi_{y}(t, \nu(t))=\frac{\chi^{\prime}(\nu(t))}{1-\chi(\nu(t))}+\phi_{y y}(t, \nu(t)) \nu^{\prime}(t),
$$

so $\nu^{\prime}(t)>0$ when $t>0$. This concludes the proof of Proposition 2.1.

We now have to show that the integral (1.18) is infinity for $t>0$. 
Lemma 2.2. Let $\phi, \nu, v$ and $\Omega^{1}$ be as in Proposition 2.1 and suppose that $u \in C^{2}\left(\Omega^{1}\right)$ satisfy $(2.1)-(2.2)$ in $\Omega^{1}$. Then

$$
\begin{aligned}
\int_{\mu(t)}^{2-t} a_{t}\left(x^{1}\right) & \left(\left(\partial_{x^{1}}-\partial_{t}\right)^{2} u\left(t, x^{1}\right)\right)^{2} d x^{1} \\
= & \int_{\nu(t)}^{\nu^{*}(t)} a_{t}(\phi(t, y))\left(\chi^{(2-l)}(y)\right)^{2} \frac{1}{\phi_{y}(t, y)} \frac{d y}{(1-v(t, \phi(t, y)))^{2}}
\end{aligned}
$$

Proof. Using the coordinates (2.11)-(2.14), we obtain

$$
U_{q q}(s, h(s, y))=\frac{1}{h_{y}(s, y)} \frac{d}{d y} U_{q}(s, h(s, y))=\frac{1}{h_{y}(s, y)} \frac{d}{d y} U_{q}(y, y)=\frac{\chi^{(2-\ell)}(y)}{h_{y}(s, y)},
$$

which, together with (2.18), proves that

$$
\left(\left(\partial_{x^{1}}-\partial_{t}\right)^{2} u\right)(t, \phi(t, y))=\frac{\chi^{(2-\ell)}(y)}{\phi_{y}(t, y)(1-v(t, \phi(t, y)))} .
$$

(2.19) follows if we use (2.20) and change variables $x^{1}=\phi(t, y), d x^{1}=\phi_{y}(t, y) d y$.

Since $\phi_{y}(t, \nu(t))=0$ and $|v|<\varepsilon<1$ it will follow that the integral (2.19) is infinity for $t>0$. In view of (1.16)-(1.17) we have

$$
a_{0}(\phi(0, y))=a_{0}(y)=\int_{x^{2}{ }^{2}+x^{3} \leq 1-(1-y)^{2}} d x^{2} d x^{3}=\pi\left(2 y-y^{2}\right),
$$

and we will prove in Proposition 2.4 below there is a constant $c>0$ such that

$$
a_{t}(\phi(t, y)) \geq c y \quad \text { if } \quad 0 \leq y \leq 1 / 4, \quad 0 \leq t<1 / 16,
$$

Assuming (2.22) for a while, the proof of that the integral (2.19) is infinity now follows from:

Lemma 2.3. There is $\chi$ satisfying the assumtions in Proposition 2.1 such that

$$
\begin{gathered}
\int_{0}^{2} y\left(\chi^{\prime \prime}(y)\right)^{2} d y<\infty \\
\int_{\nu(t)}^{\nu^{*}(t)} y\left(\chi^{(2-l)}(y)\right)^{2} \frac{d y}{\phi_{y}(t, y)}=\infty, \quad t>0 .
\end{gathered}
$$

Proof. Let us first deal with the case $\ell=0$. It is then easy to choose $\chi$ so the integral (2.23) is finite but (2.24) is infinity. We claim that

$$
\chi(y)=-C \int_{0}^{y} \varepsilon\left|\log \left(w /\left(1+w^{2 / \alpha}\right)^{\alpha / 2}\right)\right|^{\alpha} d w, \quad 0<\alpha<\frac{1}{2},
$$


will do. Note that $y \chi^{\prime \prime}(y)\left|\chi^{\prime}(y)\right|^{1 / \alpha-1}=(C \varepsilon)^{1 / \alpha} /\left(1+y^{2 / \alpha}\right)$. By $(2.9),(2.24)$ is bounded from below by

$$
\begin{aligned}
& \int_{0}^{\nu^{*}(t)} y \chi^{\prime \prime}(y)^{2} e^{-2 t \chi^{\prime}(y)} d y=\int_{b_{\varepsilon}}^{\infty} y \chi^{\prime \prime}(y) e^{2 t z} d z \geq \\
& \qquad \int_{b_{\varepsilon}}^{\infty} \frac{c_{\varepsilon} e^{2 t z}}{|z|^{1 / \alpha-1}} d z=\infty, \quad b_{\varepsilon}>0,
\end{aligned}
$$

where we have made the change of variables $z=-\chi^{\prime}(y), d z=-\chi^{\prime \prime}(y) d y$. From the same calculation when $t=0$ it follows that the integral (2.23) is finite.

If $\ell=1$ then by $(2.10),(2.24)$ is bounded from below by a constant times

$$
\int_{\nu(t)}^{\nu^{*}(t)} \frac{y\left(\chi^{\prime}(y)\right)^{2} d y}{M(t)^{2}(y-\nu(t))}=\infty
$$

It only remains to prove (2.22);

Proposition 2.4. Let $v, \phi$ and $\Omega^{1}$ be as in Proposition 2.1 and assume in addition that $|v| \leq \varepsilon=1 / 8$. Let $\Omega \subset\left\{(t, x) ;\left(t, x^{1}\right) \in \Omega^{1}, t<1 / 16\right\}$ be the largest domain of dependence for the metric whose inverse is given by

$$
\sum_{i, j=0}^{3} g^{i j}(t, x) \partial_{x^{i}} \partial_{x^{j}}=\partial_{t}^{2}-\sum_{i=1}^{3} \partial_{x^{i}}^{2}-v\left(t, x^{1}\right)\left(\partial_{t}-\partial_{x^{1}}\right)^{2}
$$

such that $\Omega \cap\{0\} \times \mathbb{R}^{3}=\left\{x ;\left(x^{1}-1\right)^{2}+x^{2}+x^{3}<1\right\}$. Then

$$
\Omega=\left\{(t, x) ;\left(t, x^{1}\right) \in \Omega^{1} \text { and } x^{2}{ }^{2}+x^{3}<D\left(t, x^{1}\right)\right\},
$$

where

$$
D(t, \phi(t, y)) \geq \frac{1}{4} y, \quad \text { when } 0 \leq y \leq \frac{1}{4}, \quad 0 \leq t \leq \frac{1}{16}
$$

Proof of Proposition 2.4. It follows from Definition 1.6 that $\left(t^{\prime}, x^{\prime}\right) \in \Omega$ if and only if $\left(t^{\prime}, x^{1^{\prime}}\right) \in \Omega^{1}$ and all Lipschitz continuous curves from $\left(t^{\prime}, x^{\prime}\right)$ that satisfy (1.8) intersect the hyperplane $t=0$ in the ball $\left\{x ;\left(x^{1}-1\right)^{2}+x^{2}{ }^{2}+x^{3}<1\right\}$. Hence Proposition 2.4 follows from Lemma 2.6 below.

Now recall Definition 1.6 of domain of dependence. An easy calculation shows that if $g^{i j}$ is given by (2.25) then the inverse satisfies

$$
\sum_{i, j=0}^{3} g_{i j}(t, x) \frac{d x^{i}}{d s} \frac{d x^{j}}{d s}=\left(\frac{d x^{0}}{d s}\right)^{2}-\sum_{i=1}^{3}\left(\frac{d x^{i}}{d s}\right)^{2}+v\left(t, x^{1}\right)\left(\frac{d x^{0}}{d s}+\frac{d x^{1}}{d s}\right)^{2}
$$


Lemma 2.5. Let $\left(t(s), x^{1}(s), x^{2}(s), x^{3}(s)\right)$ be a Lipschitz continuous curve parameterized so that $t(s)+x^{1}(s)=s$ and let $g_{i j}$ be given by (2.28). Then

$$
\sum_{i, j=0}^{3} g_{i j}(t, x) \frac{d x^{i}}{d s} \frac{d x^{j}}{d s} \geq 0, \quad x^{0}=t
$$

is equivalent to

$$
\frac{d q(s)}{d s} \leq-R(s)+v\left(t(s), x^{1}(s)\right)
$$

where

$$
q(s)=x^{1}(s)-t(s), \quad \text { and } \quad R(s)=\left(\frac{d x^{2}(s)}{d s}\right)^{2}+\left(\frac{d x^{3}(s)}{d s}\right)^{2} .
$$

Proof. The proof just follows from factoring (2.28):

$$
\begin{aligned}
\left(\frac{d x^{0}}{d s}-\frac{d x^{1}}{d s}+v\left(\frac{d x^{0}}{d s}+\frac{d x^{1}}{d s}\right)\right)\left(\frac{d x^{0}}{d s}+\frac{d x^{1}}{d s}\right)-\left(\frac{d x^{2}}{d s}\right)^{2} & -\left(\frac{d x^{3}}{d s}\right)^{2}= \\
& -\frac{d q}{d s}+v-R
\end{aligned}
$$

Lemma 2.6. Assume that $V(s, q) \in C\left(\overline{\tilde{\Omega}}_{1}\right)$ and $V_{q} \leq 0$. Suppose that $h(s, y)$ satisfies

$$
\frac{d h(s, y)}{d s}=V(s, h(s, y)), \quad h(y, y)=y
$$

and consider a curve as in Lemma 2.5 satisfying $(2.30)$ with $q(b)=h(b, y)$ for some $b>y>0$ and let $r(s)=\sqrt{x^{2}(s)^{2}+x^{3}(s)^{2}}$. Then

$$
q(a) \geq h(a, y) \quad \text { and } \quad|r(a)-r(b)|^{2} \leq|q(a)-h(a, y)||b-a|, \quad a \leq b .
$$

Suppose in addition that $r(a)^{2}=2 a-a^{2}$ when $q(a)=a$ and $|V| \leq 1 / 8$. Then

$$
r(b) \geq \sqrt{y} / 2, \quad \text { when } \quad 0 \leq t=(b-h(b, y)) / 2 \leq 1 / 8 \quad \text { and } \quad 0<y<1 / 2 .
$$

Proof. Subtracting (2.32) from (2.30) gives

$$
\frac{d(q(s)-h(s, y))}{d s} \leq-R(s)+V(s, q(s))-V(s, h(s, y)) .
$$


Since $R(s)>0$ and $q(b)=h(b, y)$ it follows by continuity that $q(s)-h(s, y)>0$ when $s<b$. Hence, after integration using that $V_{q} \leq 0$,

$$
\int_{a}^{b} R(s) d s \leq q(a)-h(a, y)
$$

since

$$
|r(b)-r(a)| \leq \int_{a}^{b} \sqrt{R(s)} d s \leq \sqrt{b-a} \sqrt{\int_{a}^{b} R(s) d s .}
$$

This proves (2.33). If we pick $a$ so that $q(a)=a$ then $a \geq y$ and

$$
q(a)-h(a, y)=a-y+\int_{y}^{a} V\left(s, h(s, y) d s \leq 9(a-y) / 8 \leq 9 a / 8 \leq 9 r(a)^{2} / 8\right.
$$

since $r(a)^{2} \geq a$ when $a \leq 1$. (2.34) follows from (2.33) since $b-a \leq b-y \leq 16 t / 7$,

$$
b-y=2 t+h(b, y)-h(y, y)=2 t+\int_{y}^{b} V(s, h(s, y)) d s \leq 2 t+(b-y) / 8 .
$$

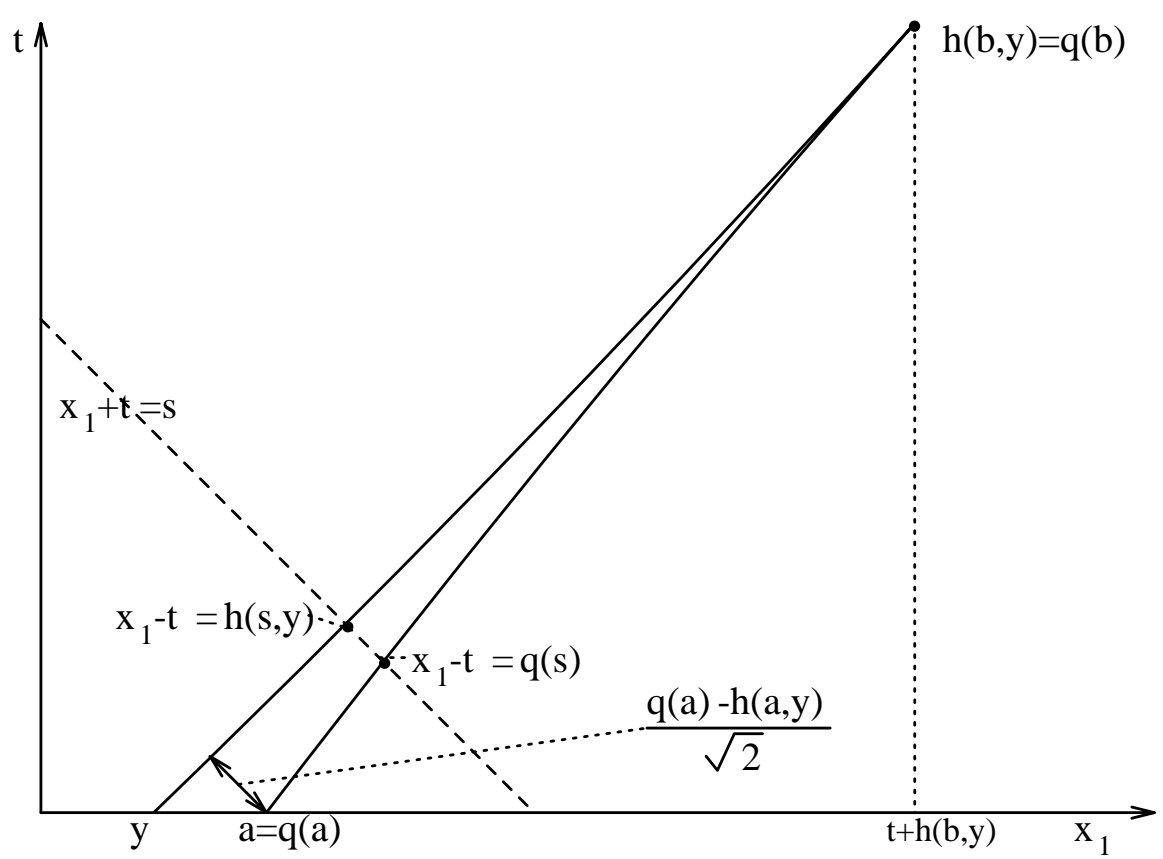

Figure 3.1 


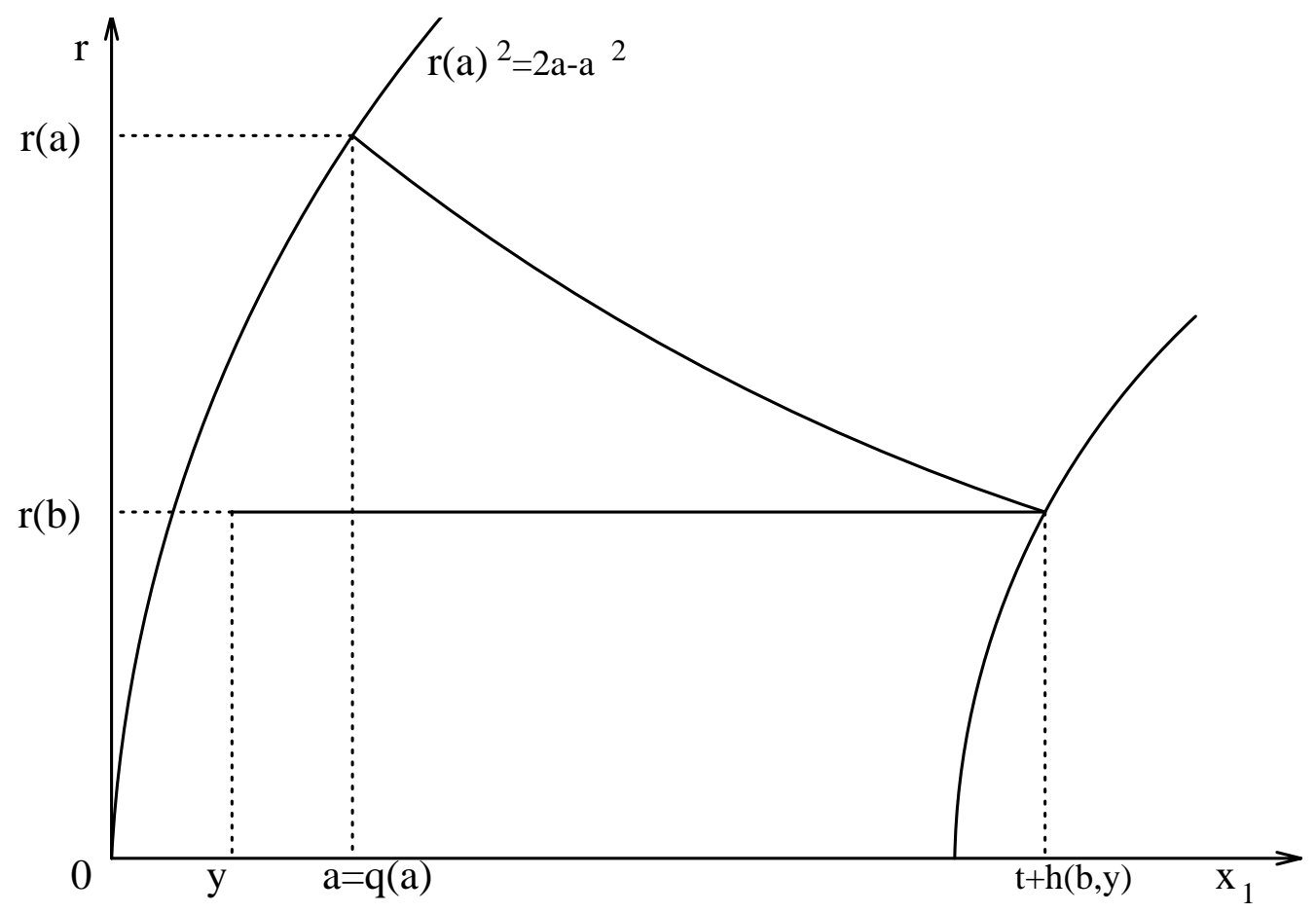

Figure 3.2

\section{Appendix I: Proof of Lemma $\mathbf{1 . 7}$}

First we note that Defintion 1.6 has two equivalent formulations:

Lemma 3.1. If $\Omega$ is a domain of dependence and $\left(t^{\prime}, x^{\prime}\right) \in \Omega$ then there is an $\varepsilon>0$ such that the closure of the cone $\Lambda_{t^{\prime} x^{\prime}}^{\varepsilon}$ is contained in $\Omega$. Here $\Lambda_{t^{\prime} x^{\prime}}^{\varepsilon} \geq \Lambda_{t^{\prime} x^{\prime}}$ is the cone cosisting of all points that can be joined to $\left(t^{\prime}, x^{\prime}\right)$ by a smooth curve $(t, x(t)) \in \Omega, t \leq t^{\prime}$, satisfying

$$
g_{i j}(x) \frac{d x^{i}}{d t} \frac{d x^{j}}{d t} \geq-\varepsilon, \quad x^{0}(t)=t
$$

Proof. Suppose that $x_{n}(t)$ is a sequence of curves satisfying (3.1) with $\varepsilon=1 / n$. Then there is a constant $C$ such that $\left|d x_{n} / d t\right| \leq C$ for all $n$. Hence we can pick a subsequence, which we also denote by $x_{n}$, such that $x_{n}(t) \rightarrow x(t)$ uniformly, where $x(t)$ is Lipschitz continuous satisfying satisfying $|d x / d t| \leq C$. We claim that $x(t)$ satisfies (3.1) with $\varepsilon=0$. This follows since $g_{i j}\left(x_{n}(t)\right) \rightarrow g_{i j}(x(t))$ uniformely and the set of vectors satisfying $g_{i j}(x(t)) Y^{i} Y^{j} \geq-\varepsilon Y^{0^{2}}$, with $Y^{0}>0$ is a convex cone.

Lemma 3.2. Let $\Omega$ is a domain of dependence and $\left(t^{\prime}, x^{\prime}\right) \in \Omega$. Then there is a smooth function $\phi$ such that with $\mathcal{H}=\{(t, x) ; t<\phi(x)\}$ we have that 
$\left(t^{\prime}, x^{\prime}\right) \in \partial \mathcal{H} \subset \Omega$ and $\partial \mathcal{H}$ is space like,

$$
\text { i.e., } \quad g^{i j}(t, x) N_{i}(x) N_{j}(x)>0 \quad \text { if } \quad t=\phi(x), \quad N(x)=\left(1,-\nabla_{x} \phi(x)\right) .
$$

Proof. Pick $t^{\prime \prime}>t^{\prime}$ such that $\left(t^{\prime \prime}, x^{\prime}\right) \in \Omega$ and $\Lambda_{t^{\prime \prime} x^{\prime}}^{\varepsilon}$ as in Lemma 3.1. Note that $\Lambda_{t^{\prime \prime} x^{\prime}}^{\varepsilon}=\left\{(t, x) ; t \leq \phi_{\varepsilon}(x)\right\}$ where $\phi_{\varepsilon}(x)=\sup \left\{t ;(t, x) \in \Lambda_{t^{\prime \prime} x^{\prime}}^{\varepsilon}\right\}$ is Lipschitz continuous. In fact if $(t, x) \in \partial \Lambda_{t^{\prime \prime} x^{\prime}}^{\varepsilon}$ then the cone consisting of all line segments from $(t, x)$ with length less than $\delta$ and slope satisfying $g_{i j}(t, x) Y^{i} Y^{j} \geq-\varepsilon Y^{0^{2}} / 2$ lies in $\Lambda_{t^{\prime \prime} x^{\prime}}^{\varepsilon}$. It follows that $\phi_{\varepsilon}$ is Lipschitz continuous and satisfies (3.2) with 0 in the right hand side replaced by $\varepsilon / 4$, if $\varepsilon$ is sufficiently small. The same then is true for $\bar{\phi}_{\varepsilon}(x)=\min \left\{\phi_{\varepsilon}(x), t^{\prime}\right\}$. By regularizing $\phi=\bar{\phi}_{\varepsilon} * \psi_{\delta}$, where $\psi_{\delta}(x)=\psi(x / \delta) / \delta^{3}, \psi \in C_{0}^{\infty}, \int \psi d x=1$, we get a smooth function satisfying (3.2) which is arbitrarily close to $\bar{\phi}_{\varepsilon}$ for which (3.2) hold and $\phi\left(x^{\prime}\right)=t^{\prime}$ if $\delta$ is sufficiently small.

To prove Lemma 1.7, we must show that in any compact subset $K \subset \Omega \cap$ $[0, T) \times \mathbb{R}^{3} u_{\varepsilon}$ tends the solution $u$ in Theorem 1.8 . Since every compact compact subset of $\Omega$ is contained in the interior of a union of sets $\mathcal{H}$ as in Lemma 3.2, it suffices to prove Lemma 1.7 for these. Let

$$
\begin{aligned}
E_{N}(w, t) & =\sup _{0 \leq s \leq t}\left(\sum_{|\alpha| \leq N}\left\|\partial^{\alpha} w(t, \cdot)\right\|_{L^{2}\left(\mathcal{H}_{s}\right)}^{2}\right)^{1 / 2}, \\
I_{N}(w, t) & =\sup _{0 \leq s \leq t} \sum_{|\beta| \leq N} \sup _{x \in \mathcal{H}_{s}}\left|\partial^{\beta} w(t, x)\right|,
\end{aligned}
$$

where $\mathcal{H}_{s}=\{x ;(s, x) \in \mathcal{H}\}$. Since $u$ is smooth in $\overline{\mathcal{H}}$ it follows that we have the bounds

$$
E_{M}(u, t) \leq K_{M}, \quad I_{M}(u, t) \leq K_{M},
$$

for any $M$. By assumption we also have

$$
I_{l}(u, t) \leq \delta .
$$

We need the following version of Sobolev type lemma:

Lemma 3.3. Suppose that $(t, x) \in \overline{\mathcal{H}}$ and $t \leq 1$. Then

$$
|w(t, x)| \leq C \sup _{0 \leq s \leq t} \sum_{|\alpha| \leq 3}\left\|\partial^{\alpha} w(s, \cdot)\right\|_{L^{2}\left(\mathcal{H}_{s}\right)},
$$

where $C$ depends on $\mathcal{H}$ but is independent of $(t, x)$.

Proof. First we will prove that

$$
\sup _{|y-x| \leq t / 2} \sum_{|\alpha| \leq 1} t^{|\alpha|}\left|\partial^{\alpha} w(0, y)\right|+C \sqrt{t} \sup _{0 \leq s \leq t} \sum_{|\alpha|=2}\left\|\partial^{\alpha} w(s, \cdot)\right\|_{L^{2}(|y-x| \leq(t-s) / 2,)} .
$$


Let $h(\tau, y)=w(t-\tau, x-\tau y)$. Then integrating by parts twice gives us the following identity

$$
\int_{0}^{t} \tau h_{\tau \tau}(\tau, y) d \tau=t h_{\tau}(t, y)-h(t, y)+h(0, y) .
$$

Since $w(t, x)=h(0,0)=h(0, y)$ we get

$$
w(t, x) \int_{|y| \leq \frac{1}{2}} d y=\int_{0}^{t} \int_{|y| \leq \frac{1}{2}} h_{\tau \tau}(\tau, y) \tau d y d \tau+\int_{|y| \leq \frac{1}{2}}\left(h(t, y)-t h_{\tau}(t, y)\right) d y .
$$

Here

$$
\begin{gathered}
\int_{0}^{t} \int_{|y| \leq \frac{1}{2}} h_{\tau \tau}(\tau, y) \tau d y d \tau \leq C \int_{0}^{t}\left(\int_{|y| \leq \frac{1}{2}} h_{\tau \tau}(\tau, y)^{2} \tau^{3} d y\right)^{\frac{1}{2}} \frac{d \tau}{\sqrt{\tau}} \leq \\
C \int_{0}^{t}\left(\int_{|y| \leq \frac{\tau}{2}} h_{\tau \tau}(\tau, z / \tau)^{2} d z\right)^{\frac{1}{2}} \frac{d \tau}{\sqrt{\tau}} \\
\leq C \sqrt{t} \sum_{|\alpha|=2} \sup _{0 \leq \tau \leq t}\left(\int_{|z| \leq \frac{\tau}{2}}\left|\partial^{\alpha} w(t-\tau, x-z)\right|^{2} d z\right)^{\frac{1}{2}} .
\end{gathered}
$$

Since $h(t, y)=w(0, x-t y)$ and $h_{\tau}(t, y)=-w_{t}(0, x-t y)-y \cdot \nabla w(0, x-t y)$, (3.5) follows. Since the conce $\{(s, y) ;|y-x| \leq(t-s) / 2\} \subset \mathcal{H}$ it follows that the second term in the right hand side of (3.5) is bounded by the right hand side of (3.4). In order to bound the first term in the right hand of (3.5) we use Sobolev Lemma for $\mathcal{H}_{0}$.

In the semilinear case we have

$$
\square \partial^{\alpha}\left(u_{\varepsilon}-u\right)=F_{\alpha}, \quad \text { where } \quad F_{\alpha}=\partial^{\alpha}\left(D^{\ell} u_{\varepsilon} D^{k-\ell} u_{\varepsilon}-D^{\ell} u D^{k-\ell} u\right),
$$

and $\ell \leq k-\ell \leq 1$. Note that $F_{\alpha}$ is a sum of terms of the form

$$
\partial^{\beta_{1}}\left(u_{\varepsilon}-u\right) \partial^{\beta_{2}}\left(u_{\varepsilon}-u\right), \quad \partial^{\beta_{1}}(u) \partial^{\beta_{2}}\left(u_{\varepsilon}-u\right), \quad \partial^{\beta_{1}}\left(u_{\varepsilon}-u\right) \partial^{\beta_{2}}(u),
$$

with $\left|\beta_{1}\right| \leq|\alpha| / 2+1$ and $\left|\beta_{2}\right| \leq|\alpha|+1$. Let

$$
m(t)=E_{N}\left(u_{\varepsilon}-u, t\right), \quad \text { and } \quad n(t)=I_{N-3}\left(u_{\varepsilon}-u, t\right),
$$

for $N \geq 8$, say. In view of (3.3) and Lemma 3.3, $n(t) \leq C m(t)$, we have

$$
\left\|F_{\alpha}(t, \cdot)\right\|_{L^{2}\left(\mathcal{H}_{t}\right)} \leq C(m(t)+C) m(t) \leq C(m(t)+C) m(t) .
$$

Recall the energy inequality;

$$
\left\|w^{\prime}(t, \cdot)\right\|_{L^{2}\left(\mathcal{H}_{t}\right)} \leq\left\|w^{\prime}(0, \cdot)\right\|_{L^{2}\left(\mathcal{H}_{0}\right)}+\int_{0}^{t}\|F(s, \cdot)\|_{L^{2}\left(\mathcal{H}_{s}\right)} d s,
$$


if $\square w=F$ and $\partial \mathcal{H}$ is spacelike. We also have the simple inequality

$$
\|w(t, \cdot)\|_{L^{2}\left(\mathcal{H}_{t}\right)} \leq\|w(0, \cdot)\|_{L^{2}\left(\mathcal{H}_{t}\right)}+\int_{0}^{t}\left\|\partial_{t} w(s, \cdot)\right\|_{L^{2}\left(\mathcal{H}_{t}\right)} d s .
$$

These two inequalities together with (3.6) implies that

$$
m(t) \leq C\left(m(0)+\int_{0}^{t}(m(s)+K) m(s) d s\right)
$$

It follows that $m(t) \leq C^{\prime} m(0)$, if $m(0)$ is sufficiently small and $t \leq 1$. By assumption $m(0) \rightarrow 0$, when $\varepsilon \rightarrow 0$. This proves that $u_{\varepsilon} \rightarrow u$ in $K$.

Let $\square_{g}=g^{i j} \partial_{i} \partial_{j}$. In the quasilinear case we have

$$
\square_{g\left(D^{\ell} u_{\varepsilon}\right)}\left(u_{\varepsilon}-u\right)=\left(\square_{g\left(D^{\ell} u_{\varepsilon}\right)}-\square_{g\left(D^{\ell} u\right)}\right) u .
$$

If $n(t)$ is sufficiently small it follows by inspection of (3.2) that $\partial \mathcal{H}$ is spacelike also with respect to the metric $g^{j k}\left(D^{\ell} u_{\varepsilon}\right)$. Assuming that this is true we will show that $n(t)$ remains small if its sufficiently small when $t=0$. Differentiating the equation gives

$$
\square_{g\left(D^{\ell} u_{\varepsilon}\right)} \partial^{\alpha}\left(u_{\varepsilon}-u\right)=F_{\alpha},
$$

where $F_{\alpha}$ satisfies the same estimate as in the semilinear case. We now have to use the energy inequality for variable coefficients $\square_{g} w=F$ that are sufficiently close to $\square$ (see Hörmander [2]),

$$
\begin{aligned}
& \left\|w^{\prime}(t, \cdot)\right\|_{L^{2}\left(\mathcal{H}_{t}\right)} \leq \\
& \quad K\left(\left\|w^{\prime}(0, \cdot)\right\|_{L^{2}\left(\mathcal{H}_{0}\right)}+\int_{0}^{t}\|F(s, \cdot)\|_{L^{2}\left(\mathcal{H}_{s}\right)} d s\right) \exp \left(\int_{0}^{t} K\left|g^{\prime}(s)\right| d s\right),
\end{aligned}
$$

where

$$
\left|g^{\prime}(s)\right|=\sum_{j, k, i} \sup _{x \in \mathcal{H}_{s}}\left|\partial_{i} g^{j k}(s, x)\right|
$$

provided that $\partial \mathcal{H}$ is spacelike and $|g|=\sum_{j, k}\left|g^{j k}-m^{j k}\right| \leq 1 / 2$. Here $m^{00}=1$, $m^{j j}=-1, j>0$ and $m^{j k}=0$ if $j \neq k$. Using this inequality we can now proceed as in the semilinear case.

\section{References}

[1] H. Bahouri and J.-Y. Chemin, Equations d'ondes quasilinears et estimations de Strichartz, preprint 1998.

[2] L. Hörmander, Lectures on Nonlinear hyperbolic differential equations, Mathmatiques \& Applications [Mathematics \& Applications], 26, Springer-Verlag, Berlin, 1997.

[3] S. Klainerman and M. Machedon, Space-time estimates for null-forms and the local existence theorem, Comm. Pure and Appl. Math. 46 (1993), 1221-1268. 
[4] S. Klainerman and M. Machedon, Finite energy solutions of the Yang-Mills equations in $\mathbf{R}^{3+1}$, Ann. of Math. 142 (1995), 39-119.

[5] S. Klainerman and M. Machedon, Smoothing estimates for null forms and applications, Duke Math. J. 81 (1996), 99-133.

[6] H. Lindblad, Global solutions for nonlinear wave equations with small initial data, Comm. Pure Appl. Math. 45 (1992), 1063-1096.

[7] H. Lindblad, A sharp counterexample to local existence of low regularity solutions to nonlinear wave equations, Duke Math. J. 72 (1993), 503-539.

[8] H. Lindblad, Counterexamples to local existence for nonlinear wave equations. Journées Equations aux Dérivées Partielles, Saint-Jean-de-Monts, Ecole Polytechnique, Palaiseau (1994).

[9] H. Lindblad, Counterexamples to local existence for semilinear wave equations, Amer. J. Math. 118 (1996), 1-16.

[10] H. Lindblad and C. D. Sogge, On existence and scattering with minimal regularity for semilinear wave equations, J. Funct. Anal. 130 (1995), 357-426.

[11] G. Ponce and T. Sideris, Local regularity of nonlinear wave equations in three space dimensions, Comm. Partial Differential Equations 18 (1993), 169-177.

[12] J. Rauch, Explosion for some semilinear wave equations, J. Differential Equations $\mathbf{7 4}$ (1988), 29-33.

[13] J. Shatah and A. Shadi Tahvildar-Zadeh, On the Cauchy Problem for equivariant wave maps, Comm. Pure Appl. Math. 47 (1994), 719-754.

[14] E. M. Stein, Singular integrals and differentiability properties of functions, Princeton Mathematical Series, No. 30, Princeton University Press, Princeton, N.J., 1970.

[15] D. Tataru, Strichartz estimates for operators with non-smooth coefficients and the nonlinear wave equation, preprint 1998.

University of California San Diego Department of Mathematics, 9500 Gilman Drive, LA Jolla, CA 92037

E-mail address: lindblad@math.ucsd.edu 